\title{
Image-guided brachytherapy (IGBT) combined with whole pelvic intensity-modulated radiotherapy (WP-IMRT) for locally advanced cervical cancer: a prospective study from Chiang Mai University Hospital, Thailand
}

\author{
Ekkasit Tharavichitkul, MD', Somsak Wanwilairat, PhD', Somvilai Chakrabandhu, MD', Pitchayaponne Klunklin, MD', \\ Wimrak Onchan, MD', Damrongsak Tippanya, BSc', Wannapa Nopnop, MSc', Razvan Galalae, MD, PhD², \\ Imjai Chitapanarux, MD \\ IThe Division of Therapeutic Radiology and Oncology, Faculty of Medicine, Chiang Mai University, Chiang Mai, Thailand, 2Faculty of Medicine. \\ Christian-Albrechts-University, Kiel, Germany
}

\begin{abstract}
Purpose: A report of preliminary results and toxicity profiles using image-guided brachytherapy (IGBT) combined with whole pelvic intensity-modulated radiation therapy (WP-IMRT) for locally advanced cervical cancer.

Material and methods: Fifteen patients with locally advanced cervical cancer were enrolled into the study. WP-IMRT was used to treat the Clinical Target Volume (CTV) with a dose of $45 \mathrm{~Gy}$ in 25 fractions. Concurrent cisplatin $\left(40 \mathrm{mg} / \mathrm{m}^{2}\right)$ was prescribed during radiotherapy (RT) on weekly basis. IGBT using computed tomography was performed at the dose of 7 Gy $\times 4$ fractions to the High-Risk Clinical Target Volume (HR-CTV).

Results: The mean cumulative doses - in terms of equivalent dose of 2 Gy (EQD2) - of IGBT plus WP-IMRT to HR-CTV, bladder, rectum, and sigmoid colon were 88.3, 85.0, 68.2 and 73.6 Gy, respectively. In comparison with standard (point A prescription) dose-volume histograms, volume-based image-guided brachytherapy improved the cumulative doses for bladder of $67 \%$, rectum of $47 \%$ and sigmoid of $46 \%$. At the median follow-up time of 14 months, the local control, metastasis-free survival and overall survival rates were $93 \%, 100 \%$ and $93 \%$, respectively. No grade $3-4$ acute and late toxicities were observed.

Conclusion: The combination of image-guided brachytherapy and intensity-modulated radiotherapy improved the dose distribution to tumor volumes and avoided overdose in OARs which could be converted in excellent local control and toxicity profiles.
\end{abstract}

Key words: brachytherapy, cervical cancer, IGBT, IMRT.

\section{Purpose}

Cervical cancer is one of the most frequently occurring cancer entities in northern Thailand. From the report of Kamnerdsupaphon et al., the age-standardized incidence rates in 2005 were 22.7 and there were 234 new cases of cervical cancer diagnosed [1]. The treatment options of cervical cancer are composed of surgery, radiotherapy and chemotherapy, according to stage and performance status of patients. Radiotherapy plays an important role in early and advanced stages of the disease. For early disease, radical radiotherapy constitutes a good option for medically operable patients. For locally advanced disease, radical radio-chemotherapy is the standard and combined modalities improve treatment results [2-4]. Radical radiotherapy composes of external beam radiotherapy (EBRT) and brachytherapy (BT). EBRT (45-50.4 Gy) aims to reduce the gross tumor and control the microscopic disease in the pelvic area, whilst brachytherapy is used to boost the dose to the local lesion up to 75-90 Gy. Although the conventional techniques yield good results, these have limitations of anatomical evaluation of tumors and organs at risk (OARs). With the incorporation of new technologies e.g. computed tomography (CT), magnetic resonance imaging (MRI), 3D planning software etc., the techniques of radiotherapy/planning have significantly improved. For 
EBRT, intensity-modulated radiation therapy (IMRT) is increasingly being used to treat cervical cancer and has the potential to improve the therapeutic ratio because of its ability to escalate the dose to cancer targets while sparing adjacent healthy tissue [5]. A few studies for IMRT in gynecological cancer showed that IMRT reduced the dose to OAR - bowels, bladder and rectum [6,7]. Respecting the principles of inverse-square law, brachytherapy delivers very high doses to the primary tumor (close to radioisotope) while it deposits substantially less dose to the surrounding OAR (bladder, rectum, and sigmoid). Although the use of conventional X-ray based planning can get good outcomes and acceptable toxicities, many limitations are associated with this process. With the emerging of Groupe Européen de Curiethérapie - European Society for Therapeutic Radiology and Oncology (GECESTRO) recommendations, the developed volume-based concept seems to be associated with an improvement of brachytherapy for the treatment of cervical cancer $[8,9]$. Many studies support the use of image-guided brachytherapy (IGBT) to improve therapeutic ratios in cervical cancer [10-12].

With the developments of radiotherapy techniques such as IMRT and IGBT, the combinations of these techniques of irradiation need to be studied. Therefore, we performed this groundwork study to report preliminary results and toxicity profiles of combined IMRT and IGBT for determining optimal treatment of cervical cancer and defining a new workflow in our department in terms of refining and improving the process quality in modern radiotherapy.

\section{Material and methods}

The study took place from January 2011 to January 2012. Seventeen patients with cervical cancer were included and treated in the Division of Therapeutic Radiology and Oncology, Faculty of Medicine, Chiang Mai University. All patients were classified IIB-IIIB by FIGO clinical staging, were between 18-70 years old and had good performance status. Patients with severe co-morbidity, emergency conditions (e.g. bleeding) that could not permit delays in treatment, pregnancy, history of previous irradiation or allergy were excluded from the study. Consent forms were signed by each patient prior to enrollment. Two patients were excluded from the study during treatment because of too large tumor extension for performing intracavitary brachytherapy, as a result fifteen patients were actually evaluated. The patient characteristic data appear in Table 1.

\section{Whole pelvic IMRT (WP-IMRT)}

All patients received EBRT of 45 Gy using IMRT. At the beginning, a CT simulation was performed. Vac-Loc was used to immobilize patients positioning. To prepare the bladder, patients were advised to urinate twenty minutes before scanning and they drank $200 \mathrm{ml}$ of water after voiding. Twenty minutes later, patients were set up in CT. This protocol was used during irradiation to maintain bladder volume. For rectal preparation, a laxative was administered in case of rectal dilatation. The pelvic region from the L1-2 interspace to the whole vagina was scanned without intravenous contrast to obtain appropriate images with the patients in supine treatment position and legs relaxed on the table. The slice thickness of CT scans was $5 \mathrm{~mm}$ without an inter-slice gap. After imaging process was completed, image data were sent to the Contouring (Oncentra Masterplan ${ }^{\circledR}$, Nucletron, an Elekta company, Elekta AB, Stockholm, Sweden) and Treatment Planning (KonRad treatment planning software ${ }^{\circledR}$, Siemens, Concord, CA, USA). For the identification of Clinical Target Volume (CTV), the RTOG/JCOG recommendations were used in combination as a guide for contouring [13-16]. The Clinical Target Volume (CTV) was composed of cervix, uterus, adnexaes, the upper half of vagina, and pelvic lymph nodes (common iliac lymph nodes [LNs], external iliac LNs, internal iliac LNs, obturator LNs and presacral LNs). Planning Target Volume (PTV) was defined by CTV plus 0.7$1.5 \mathrm{~cm}$ margin. Bladder, rectum, sigmoid colon, bowels and head of femurs were contoured as OARs. For CTV, the dose of $1.8 \mathrm{~Gy}$ per fraction, five fractions per week was prescribed to the total dose of 45 Gy. Figures 1 A-B show contours and dose distributions for WP-IMRT. The priorities were prescribed as following: CTV, bladder, rectum, sigmoid, bowel, and head of femur. The $\mathrm{D}_{90}$ (dose at $90 \%$ of volume) of CTV was calculated. For OARs, the doses at $\mathrm{D}_{2 \%}$ of bladder, rectum and sigmoid colon were recorded for dose evaluation. During irradiation, patients were advised to prepare bladder and rectum such as with CT simulations. Electronic portal imaging devices (EPID) was used weekly to evaluate and correct for actual position. The CTV and OARs doses were calculated into equivalent dose in 2 Gy-fractions (EQD2) using the linear-quadratic model and assuming $\alpha / \beta$ ratio $=10$ for tumor and $\alpha / \beta$ ratio $=3$ for OARs [17].

\section{Concurrent chemoradiation}

Concurrent chemoradiation with weekly cisplatin $40 \mathrm{mg} / \mathrm{m}^{2}$ for a maximum of five cycles was given to patients with an indication of combined radio-chemotherapy with sufficient kidney and bone marrow function. The dose of chemotherapy was modified according to a weekly assessment of creatinine clearance prior to each applied dose. Chemotherapy was interrupted when creatinine clearance was less than $40 \mathrm{ml} / \mathrm{min}$ and considered to be stopped when creatinine clearance was less than $30 \mathrm{ml} / \mathrm{min}$.

Table 1. Characteristic data of patients in the study

\begin{tabular}{lc} 
Parameters & Data (mean; range) \\
\hline Age & 53 years (range: 47-61 years) \\
\hline Pathology: & 13 \\
\hline SCCA & 2 \\
\hline ACA & 12 \\
\hline FIGO stages: & 3 \\
\hline IIB & 54 days (range: 42-74 days)
\end{tabular}

SCCA - squamous cell carcinoma, ACA - adenocarcinoma, FIGO - the International Federation of Gynecology and Obstetrics 

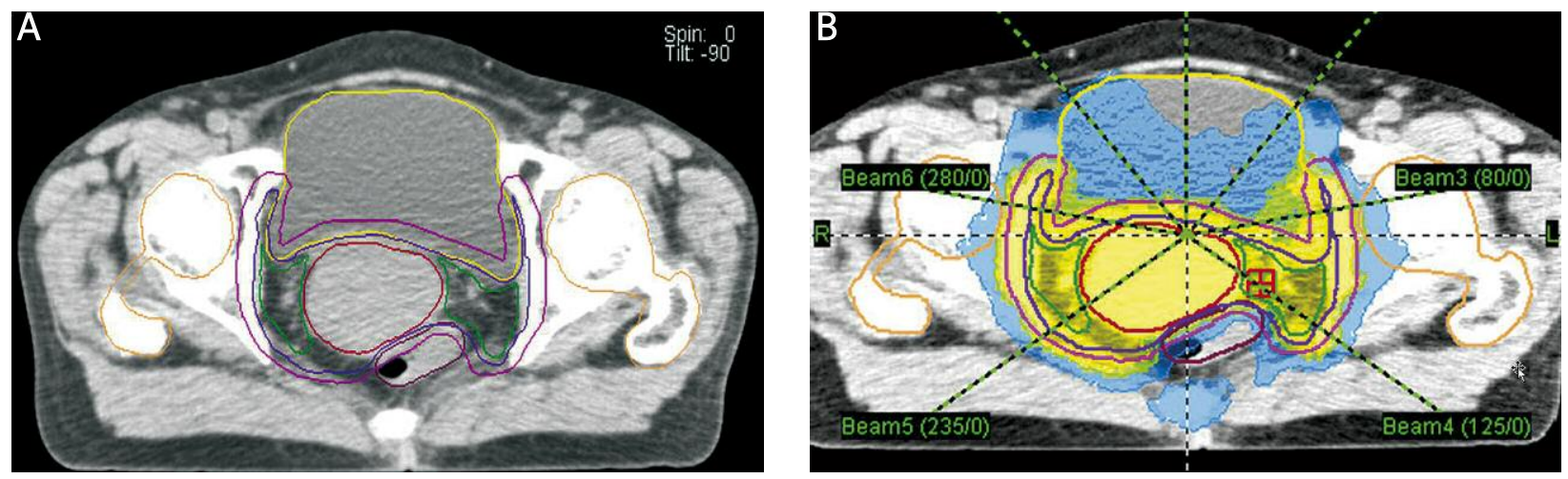

Fig. 1. Seven-beam whole pelvic intensity-modulated radiation therapy (WP-IMRT) in patient with stage IIB cervical cancer;

A) target (CTV) and OARs contouring, B) dose distribution with 45 Gy in 25 fractions

\section{Image-guided brachytherapy (IGBT)}

Four fractions of high-dose rate intracavitary brachytherapy with iridium-192 radioisotope were designed for all patients. First brachytherapy application was assigned to be performed after the $4^{\text {th }}$ week of EBRT. Intracavitary applicators were used. A Foley's catheter was inserted in the bladder and inflated with $7 \mathrm{cc}$ of diluted contrast media. A normal saline solution $(50 \mathrm{cc})$ plus $10 \mathrm{cc}$ of contrast media was added into the bladder to identify bladder volume before CT imaging. The vagina was packed with gauze to increase the distance between the radiation source and the rectum/bladder. EBRT was interrupted for each day of HDR brachytherapy insertion. After application, a pelvic CT scan was performed from the iliac crest to the ischial tuberosity without intravenous contrast to obtain appropriate images with the patients in a supine treatment position and legs relaxed on the table. The slice thickness of $\mathrm{CT}$ scans was $5 \mathrm{~mm}$ without an inter-slice gap. After the imaging was performed, images were checked by the radiation oncologist and then transported to the planning system. For the planning system, we used PLATO software (software BPS version 3.3. ${ }^{\circledR}$, Nucletron, an Elekta company, Elekta AB, Stockholm, Sweden) for the contouring and planning process. Patients were then transferred to the brachytherapy treatment room and adjusted to the same position as in the imaging devices.

High-risk clinical target volume (HR-CTV), intermediate-risk clinical target volume (IR-CTV) and OARs (bladder, rectum and sigmoid colon) were contoured according to the CT-standardized contour guidelines of Viswanathan et al. [18]. Delineation of HR-CTV was performed based on CT information at the time of brachytherapy supported by further available clinical findings. HR-CTV was identified by the volume carrying a high probability of tumor: the whole cervix and the presumed extra-cervical tumor extension at the time of brachytherapy. OARs were defined as bladder, rectum and sigmoid colon. The rectum was contoured from the anal-rectal to the recto-sigmoid junction. Delineation of the sigmoid colon started at the recto-sigmoid junction and ended $2 \mathrm{~cm}$ above the uterus. Bladder was contoured as an entire organ. Figures 2A-B show contours and dose distributions for IGBT.

The prescribed dose was at least $7 \mathrm{~Gy} \times 4$ fractions to HR-CTV (volume-based plan) for all patients. Dose-volume histograms (DVHs) to HR-CTV and OARs were evaluated. The prescription to point A was also prescribed at $7 \mathrm{~Gy}$ to evaluate DVHs as standard "point-based" plan. For HR-CTV, the $\mathrm{D}_{90}$ (minimum dose covering $90 \%$ of target volume) of HR-CTV in all patients was recorded. For OARs, the $\mathrm{D}_{2 \mathrm{cc}}$ (representing the minimum doses calculated at the most irradiated 2 cc volumes) of bladder, rectum, and sigmoid colon were recorded. The HR-CTV and OARs total doses (WP-IMRT and IGBT) were calculated into equivalent doses in 2 Gy-fractions (EQD2) using the linear-quadratic model and assuming $\alpha / \beta$ ratio $=10$ for tumor and $\alpha / \beta=3$ for OAR [17].

The EQD2 of WP-IMRT and IGBT were added to evaluate the volume-based plan with regards to the DVH constraints corresponding to a prescribed dose of at least $80 \mathrm{~Gy}_{10}$ for tumor ( $\mathrm{D}_{98 \%}$ of WP-IMRT plus $\mathrm{D}_{90 \%}$ of IGBT). For the OAR, total dose $\left(\mathrm{D}_{2} \%\right.$ of WP-IMRT plus $\mathrm{D}_{2 \mathrm{cc}}$ of IGBT)
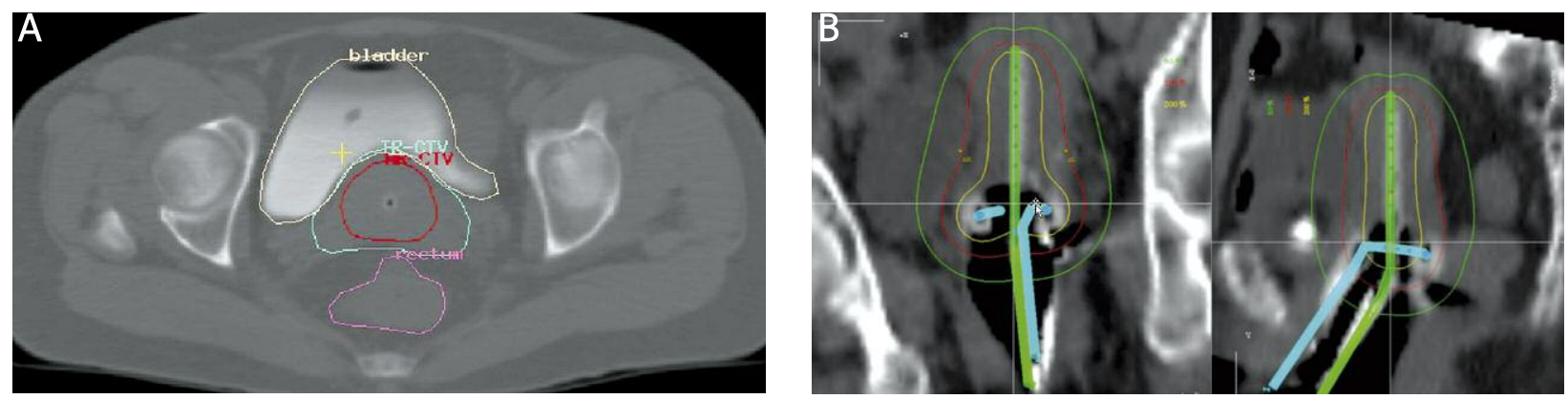

Fig. 2. Image-guided brachytherapy in patient with stage IIB cervical cancer; A) target and OARs contouring, B) dose distribution 
constraints maintained $\leq 75 \mathrm{~Gy}_{\alpha / \beta 3}$ for rectum/sigmoid and $\leq 90 \mathrm{~Gy}_{\alpha / \beta 3}$ for the bladder, respectively. For standard plans, the EQD summation of WP-IMRT of HR-CTV ( $\mathrm{D}_{98 \%}$ of WP-IMRT plus $\mathrm{D}_{90 \%}$ of HR-CTV) and OARs ( $\mathrm{D}_{2 \%}$ of WP-IMRT plus $\mathrm{D}_{2 \mathrm{cc}}$ of OARs) were calculated and compared with volume-based plans.

\section{Evaluation}

During treatment, patients visited the physician to evaluate the toxicities according to the National Cancer Institute, USA; Common Terminology Criteria of Adverse Event (CTCAE) version 3.0. After the treatment was completed, patients were appointed for a vaginal examination (PV exam) in a follow-up program. The vaginal examination was performed to evaluate the disease status according to the World Health Organization (WHO) criteria. Late toxicities were evaluated according to the Radiation Therapy Oncology Group/ European Organization of Research and Treatment of Cancer (RTOG/EORTC) late toxicity criteria.

\section{Statistical analysis}

All statistical analyses were evaluated by SPSS version 17.0. Descriptive statistics were calculated and reported at the terms of mean and range. The comparison between doses of volume-based plans versus standard (point A) plans in terms of GEC-ESTRO recommendations was performed by paired $t$-test (two-tailed test) to defined statistical significance level of $p<0.05$.

\section{Results}

All patients completed the treatment process. At median follow-up time of 14 months (range 6-18 months), one patient developed a recurrence after six months of treatment, confirmed by biopsy. One patient died from non-cancer cause six months after starting the treatment. Thus, the local control, metastasis-free survival and overall survival rates were $93 \%, 100 \%$ and $93 \%$, respectively. The volume-based IGBT planning improved dose distribution for HR-CTV and OARs in comparison with standard plans. For WP-IMRT the mean dose to $98 \%$ of CTV, $2 \%$ of bladder, $2 \%$ of rectum and $2 \%$ of sigmoid colon were $42.4 \pm 1.3 \mathrm{~Gy}, 46.7 \pm 3.2 \mathrm{~Gy}$, $46.4 \pm 4$ Gy and $48.9 \pm 3.3 \mathrm{~Gy}$, respectively. The mean volume of HR-CTV was 22.03 cc (range; 11.5-40.2 cc). Three patients $(20 \%)$ had at 1 st application a HR-CTV volume more than $30 \mathrm{cc}$. The mean cumulative EQD2 doses to HR-CTV, bladder, rectum, and sigmoid for standard plans were 109.81, $113.86,79.84$ and $90.85 \mathrm{~Gy}$, respectively. The mean cumulative EQD2 doses of volume-based plans were 88.3, 85, 68.15 and 73.64, respectively. This data is detailed in Table 2. For OARs, the volume-based planning of IGBT in comparison to standard plans could also reduce the cumulative overdose from $80 \%$ of patients $(12 / 15)$ to $13 \%$ of patients $(2 / 15)$ for bladder (> 95 Gy EQD2), from $53 \%$ of patients $(8 / 15)$ to $6 \%$ of patients $(1 / 15)$ for rectum (> 75 Gy EQD2), and from $86 \%(13 / 15)$ to $40 \%(6 / 15)$ for sigmoid colon (> $75 \mathrm{~Gy}$ EQD2). In terms of acute toxicity, nine patients (60\%) developed Grade 1-2 for proctitis and eight patients (53\%) had grade $1-2$ cystitis. Complete acute toxicity data is present- ed in Table 3. In one-year follow-up, no patient developed late toxicity except one patient with an event of Grade 1 vaginal stenosis.

\section{Discussion}

The improvement of the technology in radiotherapy has helped us to enhance the radio-therapeutic ratio. In terms of EBRT, the development of IMRT was important to be investigated. WP-IMRT was studied in combination to conventional brachytherapy in clinical aspects. The study by Portelance $e t$ al. showed that the fraction of rectal overdose with WP-IMRT was lower than conventional ( 2 or 4 fields) technique $(p<0.001)$ [19]. In the study by Hasselle et al. 101 patients were enrolled and 81 patients were treated with IMRT, followed by conventionally planned intracavitary brachytherapy. Of these patients, 63 had stage I-IIA disease and 48 had stage IIB-IVA disease. The median follow-up time was 27 months. The 3-year overall survival rate and the disease-free survival rate were $78 \%$ (95\% confidence interval [CI]: $68-88 \%$ ) and $69 \%$ (95\% CI: $59-81 \%)$, respectively. The 3-year pelvic failure rate and the distant failure rate were 14\% (95\% CI: 6-22\%) and 17\% (95\% CI: 8-25\%), respectively. Estimates of acute and late grade 3 toxicity or higher were $2 \%$ (95\% CI: 0-7\%) and 7\% (95\% CI: 2-13\%), respectively [7]. Moreover, Chen et al. reported a retrospective study using IMRT with chemotherapy in 109 patients with stage IB2-IVA cervical cancer. The dose of 45-54 Gy with IMRT technique and 20-33.5 Gy by brachytherapy at point $\mathrm{A}$ were used. At the median follow-up time of 32.5 months, the 3-year overall survival, local failure-free survival and disease-free survival rates were 78.2\%, 78.1\% and $67.6 \%$, respectively. Three patients developed grade 3 or greater acute GI toxicity and 26 patients developed grade 3 or greater hematological toxicity. Five patients de-

Table 2. Cumulative dose in EQD2 of point-based plan versus volume-based plan

\begin{tabular}{lccc} 
Parameters & $\begin{array}{c}\text { Point-based plan: } \\
\text { Gy (mean } \pm \text { SD) }\end{array}$ & $\begin{array}{c}\text { Optimized plan: } \\
\text { Gy (mean } \pm \text { SD })\end{array}$ & $\begin{array}{c}P \text {-value } \\
(\text { 2-tailed })\end{array}$ \\
\hline HR-CTV & $109.8 \pm 16.3$ & $88.3 \pm 3.8$ & $<0.001$ \\
\hline Bladder & $113.9 \pm 28.8$ & $85.0 \pm 9.3$ & $<0.001$ \\
\hline Rectum & $79.8 \pm 16.2$ & $68.2 \pm 6.5$ & 0.001 \\
\hline Sigmoid & $90.8 \pm 15$ & $73.6 \pm 6.3$ & $<0.001$
\end{tabular}

WP-IMRT - whole-pelvic intensity modulated radiation therapy, IGBT - imageguided brachytherapy, EQD2 - equivalent dose of 2 Gy, HR-CTV - high-risk clinical target volume

Table 3. Acute toxicities of cervical cancer of combination of WP-IMRT plus IGBT

\begin{tabular}{lc} 
Parameters & Numbers of patients \\
\hline Skin G1-2 & 1 \\
\hline Skin G3-4 & 0 \\
\hline Gastro intestinal toxicity (proctitis) G1-2 & 9 \\
\hline Gastro intestinal toxicity (proctitis) G3-4 & 0 \\
\hline Genitourinary toxicity (cystitis) G1-2 & 8 \\
\hline Genitourinary toxicity (cystitis) G3-4 & 0
\end{tabular}


Table 4. The published studies compared the conventional plan versus optimized plan in many methods

\begin{tabular}{|c|c|c|c|}
\hline Studies & $N$ & Methods & Results \\
\hline De Brabandere et al. [21] & 16 & $\begin{array}{l}\text { DVHs of X-ray based plan } \\
\text { versus optimization }\end{array}$ & $\begin{array}{l}\text { Overdose of the } D_{2 c c} \text { for bladder }=10 / 16 \text { and rectum }=7 / 16 \text {. } \\
\text { After optimization, no overdose was found. }\end{array}$ \\
\hline Tanderup et al. [22] & 70 & $\begin{array}{c}\text { Point doses were compared } \\
\text { to three-dimensional } \\
\text { dose parameters }\end{array}$ & $\begin{array}{l}\text { In HR-CTV }<31 \mathrm{cc} \text {, the coverage by standard plan }=94 \% \text {, } \\
\text { OARs exceeded }=72 \% \text {. } \\
\text { OARs overdose reduced to } 6 \% \text { in optimization. } \\
\text { In HR-CTV > } 31 \mathrm{cc} \text {, the coverage by standard plan = } 25 \% \\
\text { and the coverage improved to } 72 \% \text { after optimization. } \\
\text { With optimization, the coverage improved to } 72 \% \text {. }\end{array}$ \\
\hline Zwahlen et al. [23] & 20 & $\begin{array}{l}\text { Optimized plan versus } \\
\text { conventional plan }\end{array}$ & $\begin{array}{l}\text { Optimized plan improved the coverage of HR-CTV from } 70 \% \\
\text { to } 75 \% \text { and the dose to OARs could be reduced } 12-32 \% \text {. }\end{array}$ \\
\hline
\end{tabular}

Table 5. The published studies of clinical results of image-guided brachytherapy

\begin{tabular}{lccc} 
Studies & $N$ & Results & Toxicity \\
\hline Pötter et al. [11] & 156 & $\begin{array}{c}\text { 3-yr LC rate 95\%, 3-yr CSS rate 74\% } \\
\text { and 3-yr OS rate 68\% }\end{array}$ & $\begin{array}{c}\text { G3-4 bladder 3 events, G3-4 rectum 5 events, } \\
\text { G3-4 sigmoid 0 events and G3-4 vagina 2 events }\end{array}$ \\
\hline Tan et al. [12] & 28 & 3-yr CSS rate 81\% and pelvic control rate 96\% & Serious late morbidity rate 14\% \\
\hline Haie-Meder et al. [24] & 84 & 4-year OS rate 57\% and 4-year DFS rate 52\% & Four patients had grade 3 complications \\
\hline Charra-Brunaud et al. [25] & 117 & Twenty-four months local & Grade 3-4 complications 2.6\% \\
(in CCRT arm) & relapse-free survival rate 78.5\% & NA
\end{tabular}

LC - local control, CSS - cancer-specific survival, OS - overall survival, DFS - disease-free survival, yr-years

veloped grade 3 or greater chronic GI toxicity and seven patients developed grade 3 or greater GU toxicity. Although these two clinical trials used advanced technologies (IMRT) for the external beam treatment of the whole pelvis in cervical cancer with promising results, conventional brachytherapy only was used in these studies to boost the high-risk clinical target volume. [20]. In the era of IGBT, many studies reported the results of conventional versus IGBT planning approaching different technical solutions. All reports showed in conventional planning, causing inadequate dose to target volumes or overdose to OARs. With optimization, the dose to HR-CTV and OARs were improved. All studies are shown in Table 4 [21-23]. Our approach was to combine the benefits of high-technology EBRT and IGBT. Besides data concerning, dosimetry comparison between conventional and image-guided and volume-optimized brachytherapy planning, also short and long-term clinical outcomes were reported. Many studies reported the promising results of various schedules of IGBT in tumor control and late toxicities (Table 5) [10-12, 24-26].

In our experience, volume-based IGBT combined with WP-IMRT improved the dose distributions in HR-CTV and OARs compared with standard planning $[27,28]$. The mean cumulative EQD2 doses to HR-CTV could be maintained higher than 80 Gy in EQD2. For OARs, the volume-based planning of IGBT in comparison to standard plans could also reduce the cumulative dose for bladder (EQD2 113.9 vs. $85 \mathrm{~Gy}, p<0.001$ ), for rectum (EQD2 79.8 vs. $68.2 \mathrm{~Gy}, p=0.001$ ), and for sigmoid colon (EQD2 90.8 vs. $73.6 \mathrm{~Gy}, p<0.001$ ). These results demonstrate the significant potential of volume-based IGBT planning in reducing treatment related acute and late morbidity, while ensuring a sufficient cur- ative dose to HR-CTV. The mean HR-CTV in the present study was $22 \mathrm{cc}$ and thus the benefit of image-guided brachytherapy was mainly to reduce the exceeding dose to the OARs which is in concordance with the literature [22]. When considering a minimum cumulative dose of HR-CTV, all patients in the present study reached a cumulative EQD2 $>80$ Gy which represented the highest constraint priority. In the patient who developed a local recurrence, the cumulative EQD2 to the HR-CTV was high enough with $89.4 \mathrm{~Gy}$. In conclusion, our first intermediate-term results are very promising with a median follow-up time of 14 months, and disease-free survival, metastasis-free survival and overall survival rates of $93 \%, 100 \%$ and $93 \%$, respectively. No serious acute toxicity could be observed. The common toxicities were grade 1-2 proctitis (9 patients) and grade 1-2 cystitis (8 patients). Due to concurrent chemo-radiation, 5 patients $(33 \%)$ and 4 patients $(26 \%)$ had grade $1-2$ leucopenia and grade 1-2 anemia, respectively. No late toxicity was registered during the follow-up period except one patient with a grade 1 vaginal stenosis.

However, high-precision methods are more time consuming than conventional techniques which may cause more workload in the process of logistics, planning and execution of the treatment. Therefore, the summation of WP-IMRT and image-guided brachytherapy remains controversial. Further follow-up is necessary in order to evaluate the potential benefits of these sophisticated techniques in terms of long-term outcomes. The equivalent dose of $2 \mathrm{~Gy}$ (EQD2) was used in our study to calculate the dose to tumors and OARs. In this context, we assumed the summation of $\mathrm{D}_{98} \%$ of WP-IMRT (CTV) and $\mathrm{D}_{90 \%}$ of IGBT (HR-CTV) for tumor dose and the $\mathrm{D}_{2} \%$ of WP-IMRT plus $\mathrm{D}_{2 \mathrm{cc}}$ of IGBT 
for OARs doses to be significant in the evaluation of treatment quality. Prospectively, we intend to test these dosimetry measures in long-term follow-up for significance and prediction potential especially according the endpoints local control and late toxicity.

\section{Conclusion}

The combination of IGBT and WP-IMRT helped to improve significantly the target coverage, while it maintained high curative doses for HR-CTV and avoiding overdose in OARs.

\section{Acknowledgements}

The author offers many thanks to the staff of the NRUCMU in the Gynecologic Oncology Cluster and the Division of Therapeutic Radiology and Oncology, Faculty of Medicine, Chiang Mai University for supporting this study.

\section{Funding}

This work was supported by The National Research University Project (NRU) under Thailand's Office of the Higher Education Commission for financial support.

\section{References}

1. Kamnerdsupaphon P, Srisukho S, Sumitsawan Y et al. Cancers in Northern Thailand. Biomed Imaging Interv J 2008; 4: e46.

2. Rose PG, Bundy BN, Watkins EB et al. Concurrent cisplatinbased radiotherapy and chemotherapy for locally advanced cervical cancer. N Engl J Med 1999; 340: 1144-1153.

3. Keys HM, Bundy BN, Stehman FB et al. Cisplatin, radiation, and adjuvant hysterectomy compared with radiation and adjuvant hysterectomy for bulky stage IB cervical carcinoma. N Engl J Med 1999; 340: 1154-1161.

4. Eifel PJ, Winter K, Morris M et al. Pelvic irradiation with concurrent chemotherapy versus pelvic and para-aortic irradiation for high-risk cervical cancer: An update of Radiation Therapy Oncology Group trial (RTOG) 90-01. J Clin Oncol 2004; 22: 872-880.

5. Loiselle C, Koh WJ. The emerging use of IMRT for treatment of cervical cancer. J Natl Compr Canc Netw 2010; 8: 1425-1434.

6. Mundt AJ, Lujan AE, Rotmensch J et al. Intensity-modulated whole pelvic radiotherapy in women with gynecologic malignancies. Int J Radiat Oncol Biol Phys 2002; 52: 1330-1337.

7. Hasselle MD, Rose BS, Kochanski JD et al. Clinical outcomes of intensity-modulated pelvic radiation therapy for carcinoma of the cervix. Int J Radiat Oncol Biol Phys 2011; 80: 1436-1445.

8. Haie-Meder C, Pötter R, Van Limbergen E et al. Recommendations from gynaecological (GYN) GEC-ESTRO working group (I): concepts and terms in 3D image based 3D treatment planning in cervix cancer brachytherapy with emphasis on MRI assessment of GTV and CTV. Radiother Oncol 2005; 74: 235-245.

9. Pötter R, Haie-Meder C, Van Limbergen E, et al. Recommendations from gynaecological (GYN) GEC ESTRO Working Group (II): Concepts and terms in 3D image-based treatment planning in cervix cancer brachytherapy-3D dose volume parameters and aspects of 3D image-based anatomy, radiation physics, radiobiology. Radiother Oncol 2006; 78: 67-77.

10. Pötter R, Dimopoulos J, Georg P et al. Clinical impact of MRI assisted dose volume adaptation and dose escalation in brachytherapy of locally advanced cervix cancer. Radiother Oncol 2007; 83: 148-155.

11. Pötter R, Georg P, Dimopoulos JC et al. Clinical outcome of protocol based image (MRI) guided adaptive brachytherapy combined with 3D conformal radiotherapy with or without chemotherapy in patients with locally advanced cervical cancer. Radiother Oncol 2011; 100: 116-123.

12. Tan LT, Coles CE, Hart C et al. Clinical impact of computed tomography-based imaged-guided brachytherapy for cervix cancer using the tandem-ring applicator-the Addenbrooke's experience. Clin Oncol (R Coll Radiol) 2009; 21: 175-182.

13. Lim K, Small W Jr, Portelance L et al. Consensus guidelines for delineation of clinical target volume for intensity-modulated pelvic radiotherapy for the definitive treatment of cervix cancer. Int J Radiat Oncol Biol Phys 2011; 79: 348-355.

14. Taylor A, Rockall AG, Reznek RH et al. Mapping pelvic lymph nodes: guidelines for delineation in intensity-modulated radiotherapy. Int J Radiat Oncol Biol Phys 2005; 63: 1604-1612.

15. Toita T, Ohno T, Kaneyasu Y et al. A Consensus-based Guideline Defining the Clinical Target Volume for Pelvic Lymph Nodes in External Beam Radiotherapy for Uterine Cervical Cancer. Jpn J Clin Oncol 2010; 40: 456-463.

16. Toita T, Ohno T, Kaneyasu Y et al. A Consensus-based Guideline Defining Clinical Target Volume for Primary Disease in External Beam Radiotherapy for Intact Uterine Cervical Cancer. Jpn J Clin Oncol 2011; 41: 1119-1126.

17. Bentzen SM, Joiner MC. The linear-quadratic approach in clinical practice. In: Basic clinical radiobiology. Joiner M, Van Der Kogel A (eds.). $4^{\text {th }}$ ed. Edward Arnold, London 2009, pp. 120-134.

18. Viswanathan AN, Dimopoulos JC, Kirisits C et al. Computed tomography versus magnetic resonance image-based contouring in cervical cancer brachytherapy: result of a prospective trial and preliminary guidelines for standard contours. Int J Radiat Oncol Biol Phys 2007; 68: 491-498.

19. Portelance L, Chao KS, Grigsby PW et al. Intensity-modulated radiation therapy (IMRT) reduces small bowel, rectum, and bladder doses in patients with cervical cancer receiving pelvic and para-aortic irradiation. Int J Radiat Oncol Biol Phys 2001; 51: 261-266.

20. Chen CC, Lin JC, Jan JS et al. Definitive intensity-modulated radiation therapy with concurrent chemotherapy for patients with locally advanced cervical cancer. Gynecol Oncol 2011; 122: 9-13.

21. De Brabandere M, Mousa AG, Nulens A et al. Potential of dose optimisation in MRI-based PDR brachytherapy of cervix carcinoma. Radiother Oncol 2008; 88: 217-226.

22. Tanderup K, Nielsen SK, Nyvang GB et al. From point A to the sculpted pear: MR image guidance significantly improves tumour dose and sparing of organs at risk in brachytherapy of cervical cancer. Radiother Oncol 2010; 94: 173-180.

23. Zwahlen D, Jezioranski J, Chan P et al. Magnetic resonance imaging-guided intracavitary brachytherapy for cancer of the cervix. Int J Radiat Oncol Biol Phys 2009; 74: 1157-1164.

24. Haie-Meder C, Chargari C, Rey A et al. MRI-based low doserate brachytherapy experience in locally advanced cervical cancer patients initially treated by concomitant chemoradiotherapy. Radiother Oncol 2010; 96: 161-165.

25. Charra-Brunaud C, Harter V, Delannes M et al. Impact of 3D image-based PDR brachytherapy on outcome of patients treated for cervix carcinoma in France: results of the French STIC prospective study. Radiother Oncol 2012; 103: 305-313.

26. Beriwal S, Kannan N, Kim H et al. Three-dimensional high dose rate intracavitary image-guided brachytherapy for the treatment of cervical cancer using a hybrid magnetic resonance 
imaging/computed tomography approach: feasibility and early results. Clin Oncol (R Coll Radiol) 2011; 23: 685-690.

27. Tharavichitkul E, Sivasomboon C, Wanwilairat S, et al. Preliminary results of MRI-guided brachytherapy in cervical carcinoma: the Chiangmai University experience. J Radiat Res 2012; 53: 313-318.

28. Tharavichitkul E, Mayurasakorn S, Lorvidhaya V et al. Preliminary Results of Conformal Computed Tomography (CT)based Intracavitary Brachytherapy (ICBT) for Locally Advanced Cervical Cancer: A Single Institution's Experience. J Radiat Res 2011; 52: 634-640. 